\title{
Radiofrequency-assisted balloon dilatation in patients with pulmonary valve atresia and an intact ventricular septum
}

\author{
Eric Rosenthal, Shakeel A Qureshi, K Chen Chan, Robin P Martin, Douglas J Skehan, \\ Stephen C Jordan, Michael Tynan
}

\begin{abstract}
Objective-To investigate the efficacy and safety of transcatheter radiofrequency-assisted valve dilatation for infants with pulmonary valve atresia and intact ventricular septum as an alternative to the use of laser heated wires.

Design-Prospective clinical study.

Setting-Three paediatric cardiology centres.

Patients-Four children (aged 5-101 days, weight $2 \cdot 8-4 \cdot 3 \mathrm{~kg}$ ) with pulmonary valve atresia and intact ventricular septum underwent percutaneous radiofrequency-assisted valve dilatation.

Methods-After delineating the atretic valve by angiography, 0.020 inch or 0.018 inch radiofrequency wires were used to perforate the atretic valve. The valve was then dilated with conventional balloon dilatation catheters up to the valve annulus diameter.

Results-In all four cases the radiofrequency wire perforated the atretic pulmonary valve and balloon dilatation was successful. In one patient the radiofrequency wire also passed through the anterior wall of the pulmonary artery causing tamponade which required surgical repair shortly afterwards. This patient died from sepsis six days later. One patient died three weeks after the procedure from septicaemia and a paradoxical coronary embolus. Two patients were discharged after 4 and 14 days respectively.

Conclusions-Radiofrequency-assisted valve dilatation is a promising alternative to the recently developed laser wire technique. The major advantages are a reduction in cost and improved safety for the staff performing the procedure.
\end{abstract}

(Br Heart f 1993;69:347-351)

Patients with pulmonary valve atresia and intact ventricular septum present a formidable therapeutic challenge to both surgeons and cardiologists. Despite the recent improved outlook for patients with many congenital heart defects this defect continues to be associated with a high mortality. Currently the most common surgical approach to neonates and infants consists of creating a systemic to pulmonary artery shunt together with an open pulmonary valvotomy either at the same or at a later procedure. The final corrective operation entails closure of the atrial septal defect and the systemic to pulmonary shunt but can only be performed if the right ventricle develops sufficiently. Alternatively further palliative shunting procedures or a Fontan repair are required if the right ventricle remains hypoplastic. We, and others, have recently shown the feasibility of percutaneous transcatheter "hot tip" laserassisted balloon dilatation. ${ }^{12}$ In some patients this approach can replace some or all of the conventional operative stages. To overcome some disadvantages associated with the use of lasers, we have investigated the use of transcatheter radiofrequency-assisted balloon dilatation of the pulmonary valve as an alternative and report our initial experience.

\section{Patients and methods \\ PATIENTS}

Patient 1-A full term male infant with a birth weight of $2.8 \mathrm{~kg}$ became cyanosed and hypotensive a few hours after birth. After emergency resuscitation he was ventilated and prostaglandin and dobutamine infusions were started. Echocardiography confirmed pulmonary valve atresia and an intact ventricular septum and a moderate sized right ventricle. At the age of 5 days he underwent radiofrequency-assisted pulmonary valve dilatation.

Patient 2-A full term female weighing $2.67 \mathrm{~kg}$ presented with cyanosis shortly after birth. Echocardiography showed pulmonary valve atresia with an intact ventricular septum and a tripartite but hypoplastic right ventricle. A balloon atrial septostomy was performed but despite a continuous prostaglandin infusion the arterial oxygen saturation could not be maintained at a safe level and a right modified Blalock-Taussig shunt was performed. At the age of 3 months when her weight was $4.3 \mathrm{~kg}$ she was admitted for radiofrequencyassisted pulmonary valve dilatation.

Patient 3-A premature male, one of twins born at 30 weeks' gestation (weight $1.2 \mathrm{~kg}$ ) became cyanosed at birth. He was found to have pulmonary valve atresia with an intact ventricular septum and treatment with intra- 
venous prostaglandins was started. On day 32 this was changed to oral administration because of difficulty with venous access and the development of septic thrombophlebitis of the right femoral vein. He required ventilatory support until day 50 . At the age of three and a half months, when his weight was 3.7 $\mathrm{kg}$, he underwent radiofrequency-assisted pulmonary valve dilatation.

Patient 4-A full term female infant had been found to have severe pulmonary stenosis in utero. Six weeks before delivery, contrast echocardiography was performed during an attempt at pulmonary valve dilatation. This showed that the valve had become atretic but a suitable angle of approach to the pulmonary valve was not obtained and the procedure was abandoned. At birth (weight $3 \cdot 1 \mathrm{~kg}$ ) a prostaglandin infusion was started. She had radiofrequency-assisted pulmonary valve dilatation at the age of 7 days.

\section{METHODS}

Radiofrequency equipment

The 0.020 inch radiofrequency guidewire (Osypka, Germany) used in the first patient and the 0.018 inch radiofrequency guidewires used in the next three patients were developed specifically for this procedure. The 120 $\mathrm{cm}$ long insulated guidewire terminated in a 3 mm long metal tip which was heated during energy delivery. The radiofrequency guidewire was connected to a HAT 200 radiofrequency generator (Osypka) in a unipolar fashion. A large indifferent paddle was placed in contact with the patient's buttock to complete the circuit.

Before clinical use, a 0.020 inch radiofrequency guidewire was tested in a water bath at $37^{\circ} \mathrm{C}$ with fresh cadaveric pulmonary valves. Under these conditions the valves were perforated with $25 \mathrm{~W}$ of energy for 10-15 seconds.

\section{Procedure}

Under general anaesthesia the femoral artery and vein were cannulated percutaneously in patients 1,2 , and 4 . Patient 3 required a cutdown onto the left femoral vein. A $4 \mathrm{~F}$ arterial catheter was passed retrogradely through the arterial duct in patients 1,3 , and 4 or ${ }^{\text {s }}$ through the Blalock-Taussig shunt in patient 2 into the main pulmonary artery to serve as a $\vec{\omega}$ landmark. A 4 F Cobra catheter-with a $\stackrel{\omega}{\circ}$ tapered tip (Bullet catheter, Cordis, UK) in patients 1, 2, and 4 or with a 0.035 inch os lumen in patient 3-was positioned below the atretic valve (figs $1 \mathrm{~A}$ and $2 \mathrm{~A}$ ). The radiofre- $\underset{\perp}{\omega}$ quency guidewire was passed up the venous catheter into contact with the valve. $]$ Radiofrequency energy was then delivered $\vec{\nabla}$ continuously for between 3 to 5 seconds as 을. the radiofrequency guidewire was advanced. The energy setting was $25 \mathrm{~W}$ in patient 1 and 6 $5 \mathrm{~W}$ in patients 2,3 , and 4 . After the valve was penetrated the Bullet catheter was manipulated over the radiofrequency guidewire into the main pulmonary artery in
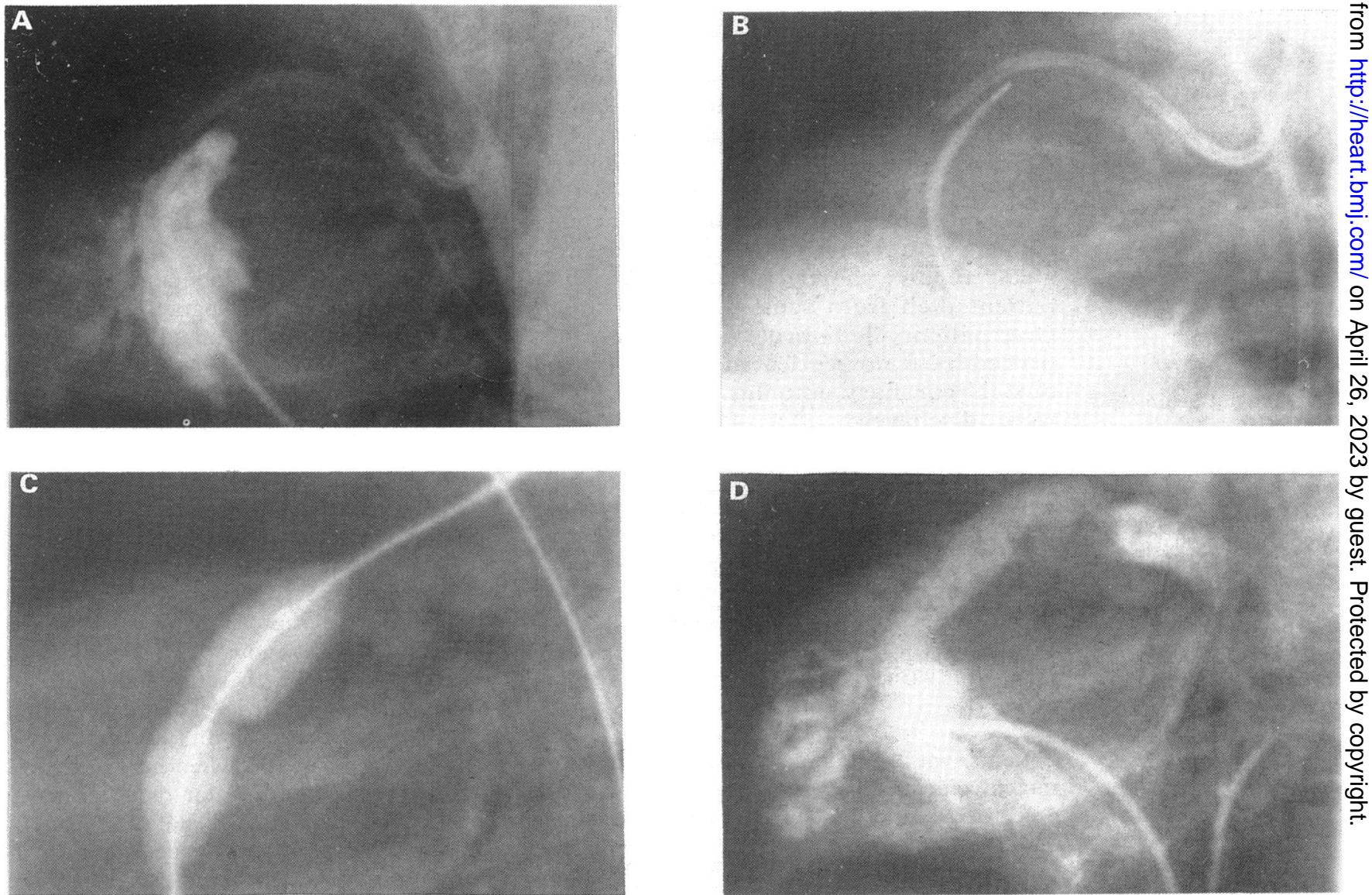

Figure 1 Right ventricular angiogram in the lateral projection with the arterial catheter indicating the course of the main pulmonary artery in patient 2 $(A)$. In $(B)$ the radiofrequency wire has crossed the valve but is not advanced as far as the marker catheter. After balloon dilation (C) angiography shows excellent flow across the pulmonary valve (D). 
Haemodynamic data ( $\mathrm{mm} \mathrm{Hg}$ ) before and after radiofrequency-assisted pulmonary valve dilatation

\begin{tabular}{|c|c|c|c|c|c|c|}
\hline \multirow{2}{*}{$\begin{array}{l}\text { Patient } \\
\text { No }\end{array}$} & \multicolumn{2}{|l|}{$P A$} & \multicolumn{2}{|l|}{$R V$} & \multicolumn{2}{|l|}{ Aorta } \\
\hline & Before & After & Before & After & Before & After \\
\hline $\begin{array}{l}1 \\
2 \\
3 \\
4\end{array}$ & $\begin{array}{l}33 / 18 \\
17 / 15 \\
40 / 20 \\
30 / 18\end{array}$ & $\begin{array}{l}\overline{22} / 12 \\
40 / 20 \\
28 / 4\end{array}$ & $\begin{array}{l}105 / 4 \\
70 / 10 \\
170 / 30 \\
120 / 5\end{array}$ & $\begin{array}{l}32 / 5 \\
22 / 10 \\
95 / 17 \\
48 / 3\end{array}$ & $\begin{array}{l}75 / 25 \\
65 / 35 \\
105 / 50 \\
75 / 20\end{array}$ & $\begin{array}{l}85 / 30 \\
80 / 40 \\
110 / 60 \\
60 / 12\end{array}$ \\
\hline
\end{tabular}

PA, pulmonary artery pressure; RV, right ventricular pressure; aorta, aortic pressure.

patients 1, 2, and 4. An exchange length floppy guidewire was then passed across the valve and snared by the arterial catheter, either in the pulmonary artery or descending aorta, to create a complete arteriovenous guidewire circuit in all the patients. In patient 3 , the radiofrequency guidewire was removed after penetrating the valve, and a balloon on a wire was passed up the catheter. It was only possible to pass the wire across the valve and balloon dilatation was unsuccessful. Therefore an exchange guidewire was passed into the main pulmonary artery and an arteriovenous guidewire circuit established. Then a Bullet catheter was used to predilatate the valve in this patient. Balloon dilatation catheters were then advanced over the guidewire circuit from the venous sheath to complete the dilatation.

\section{Results}

The valve was successfully crossed in all four patients by the radiofrequency guidewire and the valve was dilated to $8 \mathrm{~mm}$ in three patients and to $7 \mathrm{~mm}$ in patient 2 (fig $1 \mathrm{~A}-\mathrm{D}$ ). Immediate haemodynamic improvement was evident in all patients even though in patient
1 pulmonary artery pressure measurements were not obtained after the procedure (table).

Patient 2 was ventilated overnight on the intensive care unit and discharged 4 days later. Four weeks after the procedure Doppler echocardiography confirmed right ventricular to pulmonary artery flow with a velocity of $1.3 \mathrm{~m} / \mathrm{s}$ across the pulmonary valve and trivial pulmonary regurgitation. The right ventricle remained hypoplastic but the trabecular portion appeared larger. The oxygen saturation was $86 \%$.

Patient 4 was extubated two days after the procedure and treatment was changed to oral prostaglandins three days later. At two weeks the echocardiogram showed good forward flow across the pulmonary valve, with a gradient of $36 \mathrm{~mm} \mathrm{Hg}$, and that the arterial duct had closed. She was discharged with an oxygen saturation of $80 \%$. At one month the pulmonary valve gradient had increased to 50 $\mathrm{mm} \mathrm{Hg}$ and the oxygen saturation was $85 \%$.

\section{COMPLICATIONS}

In patient 1 the radiofrequency guidewire continued to advance after crossing the valve and penetrated through the anterior wall of the pulmonary artery (fig $2 \mathrm{~A}-\mathrm{D}$ ). When the radiofrequency guidewire was withdrawn into the pulmonary artery tamponade ensued but responded to needle pericardiocentesis. After an exchange guidewire was passed through the arterial duct into the descending aorta, a guidewire circuit was established, and valve dilatation performed. Shortly after completion of the procedure, tamponade recurred and required aspiration and reinfusion of the blood. The bleeding continued and at thoracotomy a $0.5 \mathrm{~mm}$ hole on the anterior aspect of the main pulmonary artery was oversewn
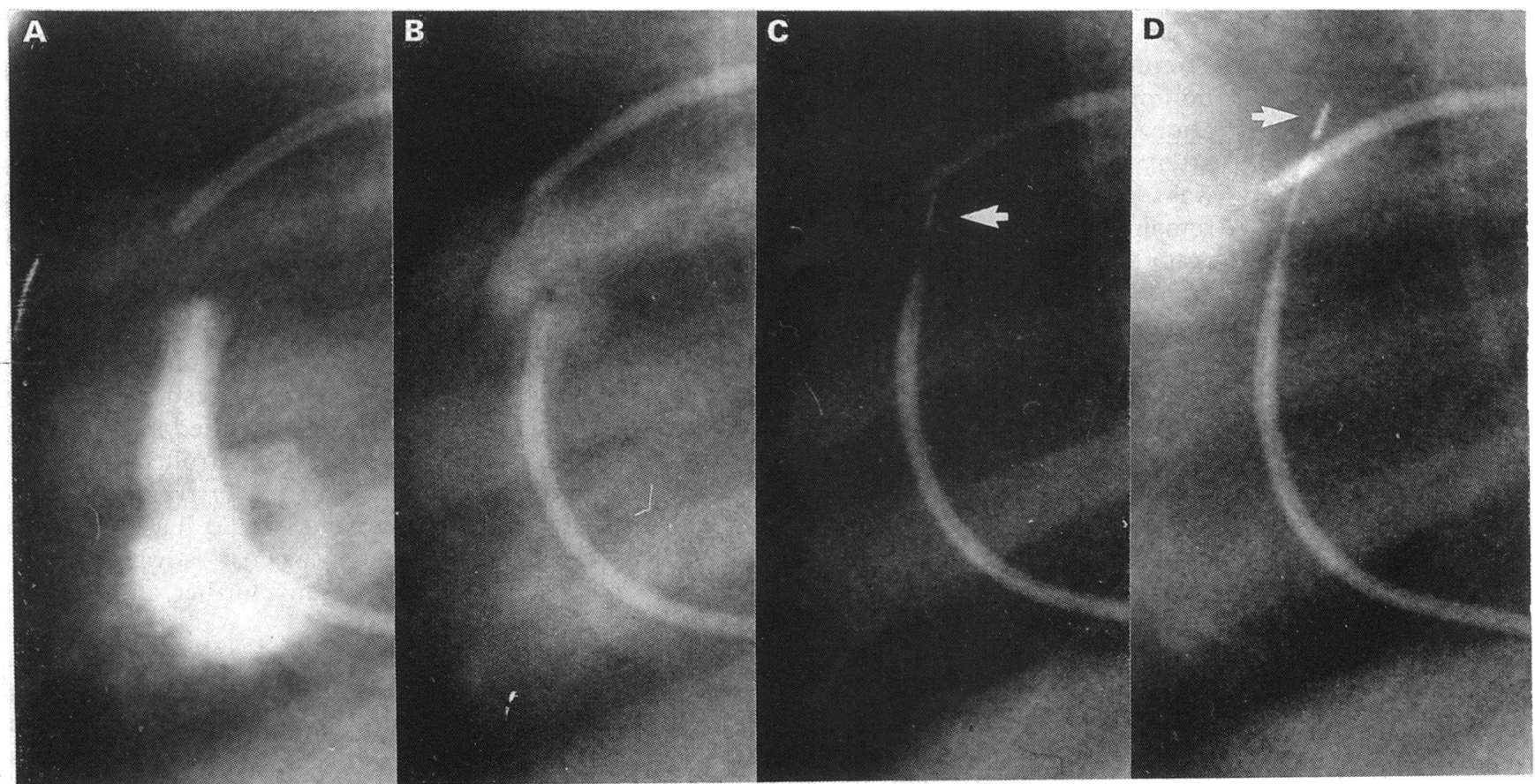

Figure 2 Right ventricular $(A)$ and pulmonary $(B)$ angiograms in the left lateral projection showing the venous catheter positioned just below the centre of the atretic valve in patient 1 . The arterial catheter in the main pulmonary artery has been passed through the arterial duct. In (C) the radiofrequency wire (arrow) has been advanced across the pulmonary valve through the venous catheter but then passes through the anterior wall of the main pulmonary artery $(D)$. 


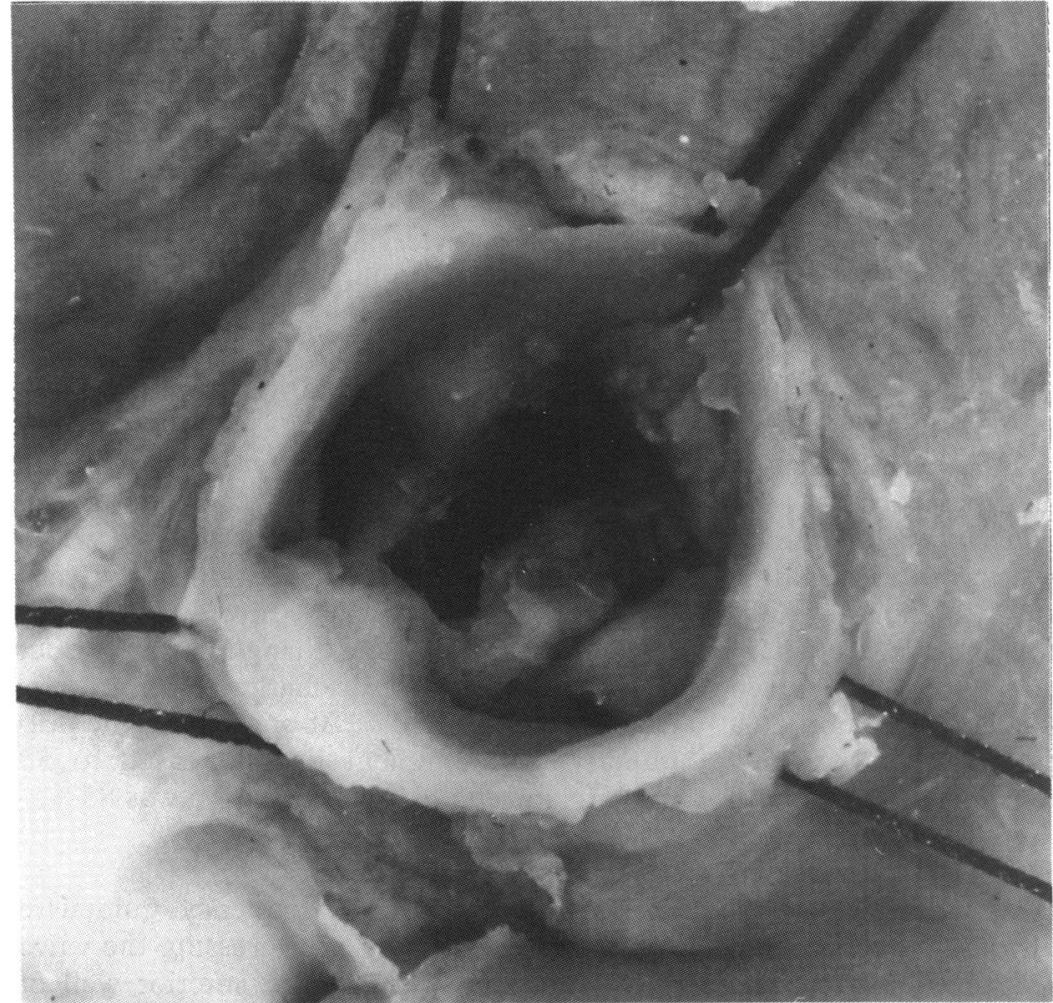

Figure 3 Postmortem examination showing that the valve has been split at two commissures producing an eccentric orifice (patient 1).

with a single stitch. When the patient returned to the intensive care unit Doppler echocardiography confirmed forward flow through the pulmonary valve with a gradient of $36 \mathrm{~mm} \mathrm{Hg}$. He became septicaemic and died 6 days later. At postmortem examination the valve was found to have a split between two commissures and there was an acceptable orifice (fig 3).

In patient 3 the right leg became pulseless after removal of the arterial catheter. Streptokinase was administered. There was no improvement in leg perfusion and at operation a clot was removed from the femoral artery. It was also noted that the femoral vein was occluded with thrombus. The leg remained ischaemic and an above knee amputation was performed two weeks later. Three days after this he suddenly became hypotensive with bradycardia and ventricular arrhythmias. He responded poorly to resuscitation and died 30 hours after this event. At postmortem examination the pulmonary valve was found to be widely patent. There was a large clot in the right atrium and a recent left ventricular infarction.

\section{Discussion}

The use of the Nd-YAG "hot tip" laser wire for percutaneous pulmonary valvotomy ${ }^{2}$ has several drawbacks. For centres without access to such a laser generator the initial capital outlay is considerable and the laser wires are relatively expensive thus increasing the procedural costs. Furthermore, most centres dealing with congenital heart disease are likely to treat no more than a handful of cases annually. There are dangers for the staff in using a powerful laser-especially the risk of retinal damage. Protective goggles have to be worn but they can be cumbersome and inconvenient in the hot environment of a paediatric interventional catheterisation laboratory. Finally, while current laser generators $\frac{\Upsilon}{\mathbb{D}}$ are far more compact than the earlier models, they are still cumbersome so that transport $c$. between laboratories and/or hospitals is time $\vec{c}$ consuming and may result in damage to the generator.

Radiofrequency heated metal tipped $\frac{\overline{\bar{N}}}{\overline{\frac{1}{}}}$ catheters have been shown to be as effective as laser heated "hot tip" catheters for recanalisation of peripheral arterial occlusions. ${ }^{3} \mathrm{We}^{\mathrm{s}}$ thus thought that use of a radiofrequency. guidewire for pulmonary valve atresia, if $\vec{\omega}$ effective, would have the advantage of reduc- $-\circ$ ing the initial costs and increasing operator comfort. Furthermore the increasing use of radiofrequency devices for the treatment of $\Rightarrow$ arrhythmias means that many institutions ${ }_{\perp}$ already have one of these generators. The ease of transfer between the electrophysio-? logical and paediatric catheterisation labora- $\vec{D}$ tories even when they are some distance aparto means that the generator can be shared.

During in vitro testing we found that the valve perforation rate was slower than with ${ }^{\omega}$ the laser wire, though with longer applications cadaver valves could be perforated at $25 \mathrm{~W}$. This led to our using greater axialo force when advancing the radiofrequency용 guidewire in our first patient, which together with the high energy setting may have con- $\overrightarrow{\overrightarrow{0}}$ tributed to the subsequent perforation of the main pulmonary artery. In addition this 0.020 inch radiofrequency guidewire was slightly stiffer than the 0.018 inch laser wire used pre- $\mathbb{\Phi}$ viously $^{12}$ or the modified radiofrequency? guidewire with a diameter of 0.018 inch used in the following three cases. We do not know: why the power setting suggested by the in $\frac{3}{3}$ vitro work was higher than that needed to perforate the valve in vivo. It is safer to begin? with a much lower setting such as $5 \mathrm{~W}$, which can be progressively increased if needed. It is important to emphasise the value of theor catheter placed in the main pulmonary artery to act as a landmark for the safe advancement ${ }_{\mathrm{W}}$ of the radiofrequency wire. In patient 2 ano unusual orientation of the main pulmonaryo artery could easily have resulted in the radiofrequency wire being aimed in the wrong.? direction. Had a more controlled advance- $\frac{T}{T}$ ment been possible in the first case the pulmonary artery catheter would have served to? warn the operator against advancing the $\cong$ radiofrequency wire any further and to stope energy application.

Radiofrequency heated guidewires offer $\frac{2}{2}$ promising alternative to the laser heated "hoto tip" guidewires for percutaneous balloon dilatation of the pulmonary valve in patients with pulmonary valve atresia. The major advantages are a reduction in cost and improved safety for the staff performing the procedure. 
We thank Jim Mackenzie of S-Pace UK for loan of the techical assistance the postmortem specimen.

ER is supported by a grant from the British Heart Foundation.

1 Qureshi SA, Rosenthal E, Tynan M, Anjos R, Baker EJ
Transcatheter laser-assisted balloon pulmonary valve dilation in pulmonic valve atresia. Am $\mathcal{F}$ Cardiol 1991; 67:428-31.

2 Parsons JM, Rees MR, Gibbs JL. Percutaneous laser valvotomy with balloon dilatation of the pulmonary valve as primary treatment for pulmonary atresia. $\mathrm{Br}$ valve as primary treatm

3 Litvack F, Grundfest WS, Papaioannou T, Mohr FW, Jakubowski AT, Forrester JS. Role of laser and thermal ablation devices in the treatment of vascular diseases. Am $\mathcal{f}$ Cardiol 1988;61:81G-6G.

\title{
ABSTRACTS IN CARDIOLOGY
}

\section{Cardiotoxicity and cholesterol}

The direct cardiotoxic effect of anthracycline (daunorubicin, doxorubicin, and epirubicin) chemotherapy is well known. Cardiotoxicity is sometimes apparent during treatment but may first present as a dilated cardiomyopathy years after completion of treatment and apparent oncological cure. Radiation treatment may also be associated with myocardial and pericardial disease which may be of very delayed onset. Raghavan et al report an absolute increase in serum cholesterol concentrations in 14 of 17 men treated with cisplatin combination chemotherapy for germ cell tumours. This finding accords with an earlier retrospective analysis of similar patients by the same workers. The exact cause of the cholesterol rise is obscure. Longterm follow up will be necessary to assess whether accelerated atherosclerosis may need to be added to the steadily increasing list of late cardiovascular effects of tumour treatment.

KAREN McLEOD

\section{Hypercholesterolaemia after chemotherapy for testis cancer}

\author{
Derk Raghavan, Keith Cox, Annabel Childs, Fohn Grygiel, David Sullivan
}

Purpose - The study was designed to determine prospectively the prevalence of fasting serum lipid abnormalities in patients who were treated with cisplatinbased chemotherapy for germ cell tumours. We unexpectedly had demonstrated hypercholesterolaemia in 20 of 30 nonfasting patients in a prior study of long-term toxicity of chemotherapy for germ cell tumours. The present study was designed to explore this phenomenon further.

Patients and methods-17 unselected patients with biopsy-proven germ cell tumours, who underwent cisplatin-based chemotherapy and who had no prior history of cardiac disease nor known hypercholesterolaemia, were studied. In addition to the standard staging tests, blood was drawn for a pretreatment fasting lipid screen, which included cholesterol, triglycerides, high-density lipoprotein (HDL) cholesterol, and apolipoproteins A1, B and (a). Repeat samples were drawn 24 hours after the administration of cis- platin and at intervals of 6 to 24 months after the completion of treatment.

Results - Seven of 17 patients ( $41 \%$ ) had higher than desirable levels of total serum cholesterol and low-density lipoprotein cholesterol. Two of them had normal levels before treatment, four had preexisting hypercholesterolaemia that increased further, and one patient had an elevated pretreatment level that did not alter. Absolute increases in serum cholesterol were noted in 14 of 17 patients. No consistent patterns of change beyond the reference ranges were found for other serum lipids.

Conclusions-We have confirmed our initial observation that serum cholesterol increases in patients who received cisplatin-containing chemotherapy regimens for germ cell tumours. Further studies will be necessary to define whether other lipid abnormalities occur and the biological significance of these findings. $(\mathcal{F}$ Clin Oncol 1992;10:1386-9.) 